\title{
A Quantitative Morphological Comparison of Cat Lingual Nerve Repair Using Epineurial Sutures or Entubulation
}

\author{
G.R. Holland ${ }^{1 *}$, D. Andrade ${ }^{2}$, K.G. Smith ${ }^{3}$, S. Lahl2 ${ }^{2}$, P.P. Robinson ${ }^{3}$, and E. Pehowich ${ }^{2}$ \\ ${ }^{1}$ Department of Cariology, Restorative Sciences and Endodontics, School of Dentistry, 1011 N. University, Ann Arbor, Michigan 48109-1078; \\ ${ }^{2}$ Department of Restorative Dentistry, Faculty of Dentistry, University of Alberta, Edmonton, Alberta T6G 2N8, Canada; and ${ }^{3}$ Department of \\ Oral \& Maxillofacial Surgery, School of Clinical Dentistry, University of Sheffield, Claremont Crescent, Sheffield S10 2TA, England; *to whom \\ correspondence should be addressed
}

\begin{abstract}
Since lingual nerves may be transected during a variety of oral surgical procedures, including third molar removal, we have investigated two possible methods of repair. Quantitative morphological observations were made on feline chorda tympani and lingual nerves proximal and distal to transection injuries repaired either by epineurial suturing or by insertion of the cut ends into a perforated silicon tube. Proximal to the repair, the most prominent difference was an increase in the number of myelinated axons in the lingual nerve following epineurial suturing but not entubulation. Proximal to the repair site, the number of nonmyelinated axons increased in comparison with controls in both chorda tympani and lingual nerves after both procedures, though the difference was statistically significant only in the lingual nerve proximal to entubulation. Distal to the injury, both types of repair showed a reduction in the number, size, and sheath thickness of myelinated axons in comparison with unoperated controls, but the difference in numbers was statistically signficant only distal to repair by entubulation. The number of non-myelinated axons distal to the repair sites was much higher than that in controls, the difference being greater distal to entubulation repair. There were more axons per Remak bundle distal to entubulation repair than to epineurial suturing, suggesting, perhaps, that fewer axons would ultimately become myelinated. Though the morphological differences between the two repair techniques are not as striking as the parallel electrophysiological differences reported previously (Smith and Robinson, 1995a,b), they are consistent with them and support the conclusion that, for transected lingual and chorda tympani nerves, epineurial suturing is the preferred approach.
\end{abstract}

Key words: lingual nerve, injury, oral surgery, taste.

Received March 7, 1995; Accepted November 16, 1995

\section{Introduction}

The removal of mandibular third molars results in temporary disturbance of lingual nerve function in up to $22 \%$ of operations (von Arx and Simpson, 1989) and permanent damage in approximately $0.5 \%$ (Mason, 1988; Blackburn and Bramley, 1989). In many patients in this latter group, it is likely that the nerve has been sectioned. In previous laboratory animal studies, we found that, three months after sectioning the combined trunk of the chorda tympani and lingual nerves, we recorded few or no responses from the chorda tympani in response to either thermal or gustatory stimuli applied to the tongue (Robinson, 1989). Vasomotor and secretomotor fibers produced smaller-than-normal responses (Robinson, 1989), and mechanosensitive fibers in the lingual branch of the trigeminal nerve were less sensitive than normal (Robinson, 1992). Morphological studies following the same injury have revealed that there is a selective loss of small myelinated fibers central to the injury, and this is consistent with the poor recovery of gustatory and chemosensitive fibers (Holland $e t$ al., 1996). We are investigating the effect of nerve repair on these physiological and morphological changes.

Several approaches to nerve repair have been developed to improve the recovery and minimize the sensory and functional disturbances following transection. They involve either bringing and holding the cut ends together or introducing a guidance material between the cut ends. The most commonly used technique for approximating the cut ends of a sectioned nerve is still epineurial suturing, but damage and scarring associated with the sutures may lead to a region in which axons become blocked or entangled into neuromas. Entubulation is an alternative technique in which the proximal and distal ends of the nerve are introduced into a tube of either resorbable or non-resorbable material, and reports have revealed very variable degrees of success (Ashur et al., 1987; Fields et al., 1989). It has the advantage of allowing gaps between the cut ends to be bridged and could permit the inclusion of neurotrophic substances. In recent electrophysiological studies, we have compared the efficacy of epineurial suturing with that of entubulation in the repair 
of sectioned feline lingual nerves (Smith and Robinson, $1995 \mathrm{a}, \mathrm{b})$. These studies revealed that the characteristics of reinnervated receptors had returned nearer to normal after epineurial suture repair. The present report describes parallel quantitative morphological findings from these experiments in the form of an ultrastructural survey of axonal populations proximal and distal to sectioned lingual nerves repaired by the two methods.

\section{Materials and methods}

\section{Animal preparation}

Thirteen adult cats, approximately 2 years of age at the time of nerve injury, were used in these experiments. The experimental protocol was approved by the Home Office (UK). The animals were anesthetized with a mixture of alphaxalone and alphadolone acetate (Saffan, Glaxo Laboratories; induction, 18 $\mathrm{mg} / \mathrm{kg}$ i.m., maintenance $2 \mathrm{mg} / \mathrm{kg}$ i.v.) and intubated; the left lingual nerve was exposed and sectioned with scissors. The surgical procedure has been described in detail elsewhere (Smith and Robinson, 1995a). The site of the lesion was 3 to 5 $\mathrm{mm}$ distal to the junction between the chorda tympani and the lingual branch of the trigeminal nerve (for simplicity, the latter will be referred to as the 'lingual' nerve). In 6 animals, the nerve was repaired with epineurial sutures of $9 / 0$ ethilon (Ethicon Ltd UK). Either 5 or 6 sutures were placed. In the remaining animals, the nerve was repaired by insertion of the nerve stumps $1.5 \mathrm{~mm}$ into a sterile silicone tube (4 mm long, $1.2 \mathrm{~mm}$ inside diameter, $2 \mathrm{~mm}$ outside diameter; Portex Ltd, Kent, UK). Three diamond-shaped holes had been cut into the walls of the tube as described by Jenq and Coggeshall (1985), and each end of the nerve was secured in the tube with a single $9 / 0$ suture, leaving a gap of $1 \mathrm{~mm}$ between the two ends (see Smith and Robinson, 1995a). The surgical site was irrigated with sterile saline and sutured closed in layers.

Twelve weeks later, with the animals under general anesthesia (sodium pentobarbitone, induction $42 \mathrm{mg} / \mathrm{kg}$ i.p., maintenance $3 \mathrm{mg} / \mathrm{kg}$ i.v.), the left lingual nerves were exposed, and both whole-nerve and single-unit recordings were made central to the repair site. These observations have been reported elsewhere (Smith and Robinson, 1995a,b). At the completion of the electrophysiological observations, the lingual nerve on both operated and control sides was dissected out and placed in fixative $(2.5 \%$ glutaraldehyde, $4 \%$ dextran $\mathrm{T}-40$ in $0.1 \mathrm{M}$ cacodylate buffer).

\section{Tissue processing}

Each nerve was then divided, processed for electron microscopy according to standard protocols (e.g., Holland and Robinson, 1990), and embedded in Araldite so as to present, from the repaired nerves, one level 3 to $5 \mathrm{~mm}$ central to the repair site and another 3 to $5 \mathrm{~mm}$ distal to it, as block faces. A sample equivalent to the level of the transection was prepared from the contralateral (control) nerve. Both semi-thin $(0.5$ to $1.0 \mu \mathrm{m})$ and ultrathin (50 to $100 \mathrm{~nm}$ ) sections of each block face were cut. The semi-thin sections were stained with toluidine blue and examined in the light microscope. The ultrathin sections were stained with uranyl acetate and lead citrate and examined in a Hitachi 7000 electron microscope.

\section{Microscopy}

Light microscopy was used to determine the number of fascicles present. The perimeter of each fascicle was traced with a camera lucida, and from this tracing the area was determined by means of a digitizing pad linked to a computer ('Bioquant' software, $R$ \& M Biometrics, Nashville, TN, USA).

A technique described by Mayhew and Sharma (1984) was used to estimate the fiber population of each nerve trunk. This method consists of counting axons in $6 \%$ of the cross-sectional area of each nerve fascicle in a systematic randomized pattern. In nerves in which the chorda tympani and lingual nerve were contained in the same fascicle, the two components were distinct and were sampled separately. The size of the sample area was determined from the light microscope measurements, and the number of grid squares required to accommodate this area on a standard 300 slim mesh grid was calculated. Allowance was made in this calculation for a 'guard area' within each grid space around the area of measurement to ensure that it was possible to include in the counts all axons that had their centers in the sample area. This would also allow for size measurements of all counted axons. The grid squares that contained axons were numbered, and the first square to be measured was determined by a random number generator. The other squares to be included were counted sequentially from the first. Each included grid square was then photographed in the electron microscope at a magnification of X1200 and printed, as a montage, at a final magnification of $X 8400$. The numbers of myelinated axons were counted and their circumference measured and the thickness of the myelin sheath determined by digitization ('Bioquant' software). The number of non-myelinated fibers (Remak bundles) was counted and the number of axons per fiber recorded.

\section{Data analysis}

Statistical comparisons between control and operated sides were made by Student's paired $t$ test. Comparisons between the two repair groups were made by Student's grouped $t$ test.

\section{Results}

\section{Light microscopy}

Control nerves (Fig. 1). The chorda/lingual nerves from the unoperated sides were similar to those we have described earlier (Holland and Robinson, 1992; Holland et al., 1995). In each case, the large myelinated axons were predominant, but a distinct group of narrower fibers, comprising the chorda tympani, was always present. The chorda tympani formed a distinct fascicle in 5 of the 13 control nerves examined. In the other 8 , the chorda tympani was within the same fascicle as the lingual nerve but was clearly recognizable as a separate group of smaller axons. When within a single fascicle, the smaller axons of the chorda tympani were usually separated from the larger 
axons by a thickening of the endoneurium. In one specimen, 4 fascicles were present with the chorda tympani present within one large fascicle along with many wide-diameter axons; the 3 other smaller fascicles contained largediameter axons. The mean number of fascicles on the control side of animals in which the injured nerve had been repaired by epineurial suturing was 2 $( \pm 1.1)$ and that from the control side of animals in which the repair was by entubulation was 1.32 ( \pm 0.52 ). The difference was not statistically signficant.

Proximal to repair site. Epineurial suture (Fig. 2).
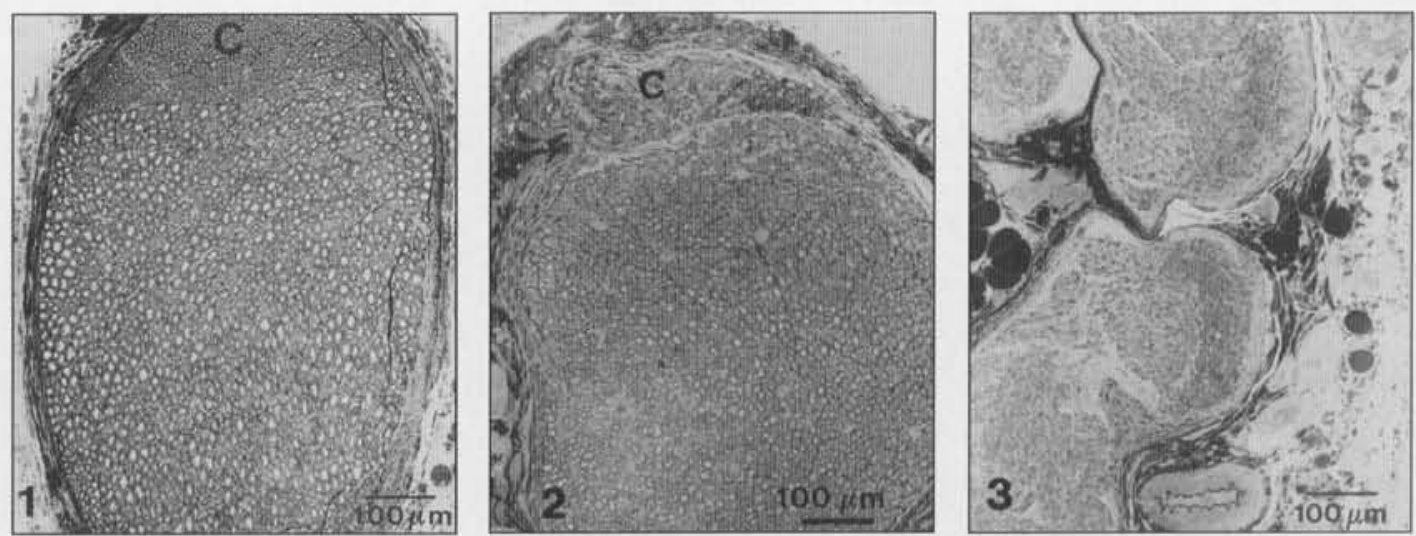

Figure 1. An unoperated control lingual/chorda tympani nerve. The combined nerve trunk is a single fascicle with the chorda tympani evident as a distinct group of smaller axons (C) in the upper part of the micrograph. Light micrograph of a semi-thin section stained with toluidine blue.

Figure 2. A lingual/chorda tympani nerve proximal to an epineurial suture repair. Chorda tympani (C) is lower part of the micrograph. At this magnification, the nerve is indistinguishable from controls. Light micrograph of a semi-thin section stained with toluidine blue.

Figure 3. The combined nerve trunk distal to an epineurial suture repair. One complete and parts of three other fascicles are visible. None is recognizable as the chorda tympani. In comparison with Fig. 1, it can be noted that the axonal sizes are generally closer in size to those of the chorda tympani than the lingual. Light micrograph of a semi-thin section stained with toluidine blue.

Four nerves had a single fascicle within which a distinct grouping of smaller axons (the chorda tympani) was visible. In the other two, the chorda tympani formed a separate fascicle. The mean number of fascicles proximal to epineurial suture repair was $1.67( \pm 0.52)$, and this was not significantly different from the controls $(p>0.05)$.

Entubulation repair. In all of the specimens, two fascicles were present. This is statistically different from controls $(\mathrm{p}=0.019)$.

Distal to repair site (Fig. 3). No distinct chorda tympani was present in any of the specimens. More fascicles were present after both types of repair ( $7.67 \pm 5.58$ after epineurial suturing; $6.29 \pm 4.11$ after entubulation) than were found in contralateral controls, and in both cases the difference is statistically significant $(p<0.05)$ from both controls and nerves proximal to the repair. The differences between fascicle counts distal to the two types of repair were not statistically significantly different.

\section{Electron microscopy}

The qualitative ultrastructural appearance of the nerve trunk proximal to both types of repair does not differ. Distal to the repair sites (Figs. 4, 5), a preponderance of non-myelinated axons is seen. Numerical data derived from the micrographs are shown in Tables 1 through 4 . For all data except the axon counts, the mean presented is computed from the mean value from each specimen rather than from each individual measurement. Since our previous examination of undamaged nerves (Holland and Robinson, 1992) has shown a lower variation in axon numbers and sizes side to side within the same animal than between animals, comparisons are shown between unoperated and operated sides. The relative degree of change due to the two procedures expressed as percentages is then summarized in Table 5.

\section{Data analysis}

\section{Proximal to the injury site}

Numbers of myelinated axons (Tables 1, 2, and 5). The mean number of myelinated axons in the chorda tympani proximal to both types of repair was reduced in comparison with controls, and this was more marked after entubulation than after suturing $(23.63 \%$ vs. $9.34 \%)$. The differences, however, were not statistically significant either between operated and control sides or between the two operated groups $(p>0.05)$. The mean number of myelinated axons in the lingual nerve proximal to injuries repaired by epineurial

Table 1. The mean number, size, and myelin thickness of myelinated axons in controls and after epineurial suture. Asterisks indicate means that are statistically significantly different from controls $(p<0.05)$.

\begin{tabular}{l|c|c|c|c|c|c|c}
\hline \multirow{2}{*}{$\begin{array}{l}\text { Suture } \\
(\mathrm{n}=6)\end{array}$} & \multicolumn{3}{|c|}{ Control } & \multicolumn{3}{c|}{ Proximal } & Distal \\
\cline { 2 - 8 } & C.T. & Lingual & Total & C.T. & Lingual & Total & Total \\
\hline No. (SD) & $1712(176)$ & $3379(132)$ & $5091(1230)$ & $1552(759)$ & $4383^{*}(971)$ & $5716(983)$ & $4751(344)$ \\
Circum., $\mu \mathrm{m}(\mathrm{SD})$ & $14.04(1.23)$ & $21.98(2.21)$ & $19.73(2.58)$ & $14.23(1.43)$ & $23.23(3.13)$ & $20.35(1.92)$ & $14.27^{*}(1.54)$ \\
Myel. sheath, $\mu \mathrm{m}(\mathrm{SD})$ & $0.71(0.08)$ & $1.13(0.09)$ & $1.00(0.10)$ & $0.69(0.10)$ & $1.14(0.16)$ & $1.00(0.11)$ & $0.67^{*}(0.04)$ \\
\hline
\end{tabular}



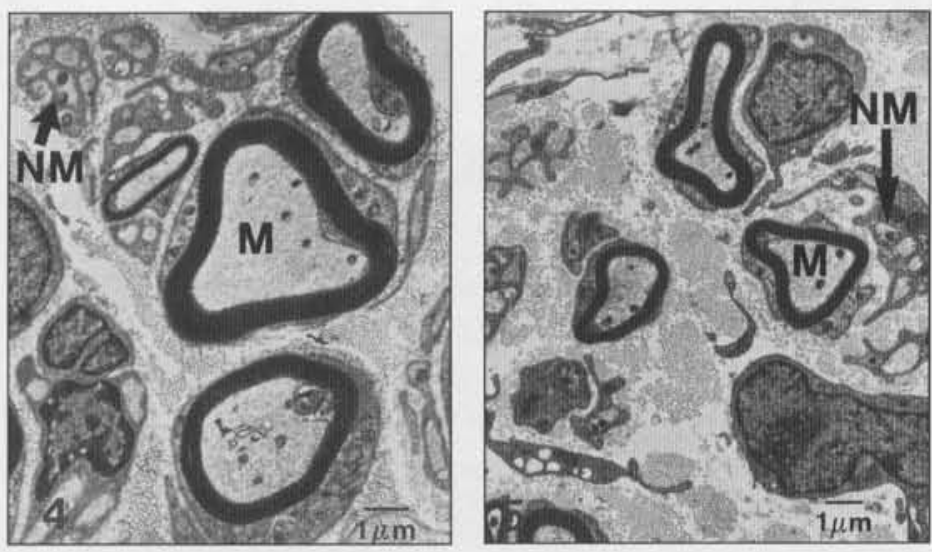

Figure 4. Myelinated (M) and non-myelinated (NM) axons from the combined nerve trunk distal to an epineurial suture repair. Electron micrograph.

Figure 5. Myelinated (M) and non-myelinated (NM) axons from the combined nerve trunk distal to an entubulation repair. Electron micrograph.

suturing was higher than that in controls $(\mathrm{p}<0.05)$ but proximal to entubulation remained close to control values.

Sizes of myelinated axons (Tables 1, 2, and 5). Mean myelinated axonal circumferences after each repair type were similar to each other and to control values $(p>0.05)$. Sheath thickness appeared to be reduced after entubulation but not after suturing by approximately $15 \%$ in both lingual and chorda tympani nerves, though the difference was not statistically significant.

Numbers of non-myelinated axons (Tables 3, 4, and 5). Proximal to both types of repair, the counts for non-myelinated axons were consistently higher for both lingual and chorda tympani nerves. The difference, however, was statistically significant only between controls and lingual nerves repaired by entubulation $(p=0.044)$. Direct comparison of counts proximal to epineurial suture repair and entubulation revealed no statistically significant difference $(p>0.05)$.

Axons per Schwann cell (Tables 3, 4, and 5). There was no significant difference in the number of axons per Schwann cell in control nerves and those proximal to the repair sites, nor between nerves proximal to the two types of repair $(p>0.05)$.

\section{Distal to the injury site}

Since it was impossible to distinguish lingual and chorda tympani components distal to either lesion, comparisons were made only on the total (chorda tympani plus lingual) counts and compounded sizes, sheath thicknesses, and Remak bundle populations.

Numbers of myelinated axons (Tables 3, 4, and 5). The mean number of myelinated axons distal to both types of repair was reduced in comparison with total counts from controls. The difference was more marked and statistically significant only distal to repair by entubulation $(p<0.01)$. The differences between counts distal to the two repairs did not differ significantly.

Sizes of myelinated axons (Tables 1, 2, and 5). Distal to both types of repair, myelinated axon sizes were reduced in comparison with controls. The reductions were similar, and in each group both were statistically significant $(p<0.01)$.

Myelin sheath thickness (Tables 1, 2, and 5). Sheath thickness was reduced by a similar degree distal to both types of repair, and both were statistically significant from controls $(\mathrm{p}<0.01)$.

Numbers of non-myelinated axons (Tables 3, 4, and 5). Both types of repair resulted in large increases in the number of nonmyelinated axons distal to the injury, and in each case the difference was statistically significant $(\mathrm{p}<0.01)$. The difference was more marked distal to entubulation ( $314 \%$ of controls) than after epineurial suturing ( $184 \%$ of controls), though this difference was not statistically significant $(p>0.05)$.

Axons per Schwann cell (Tables 3, 4, and 5). The number of axons per Schwann cell increased distal to entubulation repair but not distal to epineurial suturing. The difference distal to entubulation and suturing is statistically significant $(\mathrm{p}<0.05)$.

\section{Discussion}

The most marked change in the chorda tympani/lingual nerve at the light microscope level was the dramatic increase in the number of fascicles distal to the injury site. A similar increase (to $5.5 \pm 5.2$ ) occurred in our previous study on sectioned nerves without repair (Holland et al., 1995), and although the increase after repair appears greater, the difference is not statistically significant $(p>0.05)$. It is perhaps surprising that neither repair technique prevents the increase in the number of fascicles. Proximal to the injury site in other nerves, numerous "minifascicles" have been described (Morris et al., 1972). The proximal fascicular pattern in this study did not differ from controls after either suturing or entubulation.

Proximal to the injury in sectioned nerves that are not repaired, there appears to be a substantial and selective loss of narrow myelinated fibers which has the greatest effect on the fiber population in the chorda tympani (Holland et al.,

Table 2. The mean number, size, and myelin thickness in controls and entubulation repair. Asterisks indicate means that are statistically significantly different from controls $(\mathrm{p}<0.05)$.

\begin{tabular}{l|c|c|r|r|r|r|r}
\hline \multirow{2}{*}{$\begin{array}{l}\text { Entubul. } \\
(\mathrm{n}=7)\end{array}$} & \multicolumn{3}{|c|}{ Control } & \multicolumn{3}{c|}{ Proximal } & Distal \\
\cline { 2 - 7 } & C.T. & \multicolumn{1}{|c|}{ Lingual } & Total & C.T. & Lingual & \multicolumn{1}{c}{ Total } & Total \\
\hline No. (SD) & $1532(150)$ & $3583(705)$ & $5109(901)$ & $1170(876)$ & $3420(2040)$ & $5073(3176)$ & $4136^{*}(972)$ \\
Circum., $\mu \mathrm{m}$ (SD) & $14.83(0.96)$ & $24.12(2.03)$ & $20.93(1.45)$ & $14.99(2.44)$ & $22.48(5.70)$ & $18.91(4.69)$ & $14.23^{*}(1.58)$ \\
Myel. sheath, $\mu \mathrm{m}$ (SD) & $0.77(0.12)$ & $1.20(0.20)$ & $1.07(0.16)$ & $0.65(0.17)$ & $1.02(0.30)$ & $0.87(0.26)$ & $0.67^{*}(0.04)$ \\
\hline
\end{tabular}


Table 3. The mean number of non-myelinated axons per nerve and the mean number of axons present in each Schwann cell in control and after epineurial suture nerve repair. Asterisk indicates means that are statistically significantly different from controls $(\mathrm{p}<0.05)$.

\begin{tabular}{|c|c|c|c|c|c|c|c|}
\hline \multirow{2}{*}{$\begin{array}{l}\text { Suture } \\
(n=6)\end{array}$} & \multicolumn{3}{|c|}{ Control } & \multicolumn{3}{|c|}{ Proximal } & Distal \\
\hline & C.T. & Lingual & Total & C.T. & Lingual & Total & Total \\
\hline No. (SD) & $1217(201)$ & $3210(1343)$ & $4427(1446)$ & $1438(781)$ & $5136(2068)$ & $6573(2679)$ & $8138 *(3023)$ \\
\hline Ax./fiber (SD) & $2.08(0.29)$ & $2.67(0.59)$ & $2.44(0.39)$ & $2.19(0.47)$ & $2.65(0.59)$ & $2.48(0.49)$ & $2.09(0.38)$ \\
\hline
\end{tabular}

1996). This loss does not occur following crush injuries and is thus consistent with the difference in changes in taste and thermal sensitivity following the two types of injury (Robinson, 1989). The mean count of myelinated axons in the chorda tympani proximal to the unrepaired nerve section was $50 \%$ of controls (Holland et al., 1996). The mean count of myelinated axons in the chorda tympani proximal to epineurial suture and entubulation repair was reduced by 9.35 and $23.63 \%$, respectively, though in neither case was the difference statistically significant from controls. In addition, the mean size changes proximally in the chorda tympani are not suggestive of a significant preferential small fiber loss, as was seen in unrepaired nerves. It seems that both repair methods minimize the proximal changes to the chorda tympani but that epineurial suturing is, perhaps, the more effective in this regard.

The population of myelinated axons in the lingual nerve was increased ( $30 \%$ more than controls) proximal to suture repair sites. No similar change was seen proximal to entubulation repair. This increase is presumably due to either branching of axons central to the injury site or the presence of recurrent nerve sprouts. Although recurrent myelinated axons do occur, they are reported to be of small diameter (Aitken, 1949; Aitken and Thomas, 1962). The mean diameter proximal to suture repair in this study was not reduced in comparison with controls. Beggs et al. (1986) measured fiber densities rather than populations and, while reporting an increase in the proportion of narrower myelinated fibers, found no overall change in density. Cabaud et al. (1976) made axonal counts proximal and distal to sutured repair of cat ulnar nerves. Distal counts were $85 \pm 12 \%$ of proximal counts, but, since unoperated controls were not included, it is not clear whether this was due to a distal decrease or a proximal increase or a combination of both. The functional signficance of this change is unclear. These recurrent axons could be responsible for aberrant afferent activity and would not be available to reinnervate target tissues.

Reductions in myelination in the proximal segments of other regenerating nerves have been described (Lubinska, 1961; Aitken and Thomas, 1962). In the chorda tympani and lingual nerve, this seems to be prevented by epineurial suture repair but not by entubulation.
Table 4. The mean number of non-myelinated axons per nerve and the mean number of axons present in each Schwann cell in controls and after entubulation repair. Asterisk indicates means that are statistically significantly different from controls $(p<0.05)$.

\begin{tabular}{l|c|c|c|c|c|c|c}
\hline \multirow{2}{*}{$\begin{array}{l}\text { Entub. } \\
(\mathrm{n}=7)\end{array}$} & \multicolumn{4}{|c|}{ Control } & \multicolumn{1}{c|}{ Proximal } & Distal \\
\cline { 2 - 8 } & C.T. & Lingual & \multicolumn{1}{c|}{ Total } & C.T. & \multicolumn{1}{c|}{ Lingual } & Total & Total \\
\hline No. (SD) & $1149(503)$ & $2804(1425)$ & $3926(2035)$ & $2170(1953)$ & $5490^{*}(2615)$ & $8623(5364)$ & $12359^{*}(5100)$ \\
Ax./fiber (SD) & $2.12(0.66)$ & $2.40(0.46)$ & $2.23(0.49)$ & $2.98(0.58)$ & $2.77(0.48)$ & $2.80(0.49)$ & $2.98(0.57)$ \\
\hline
\end{tabular}

The number of nonmyelinated axons proximal to entubulation repair was significantly greater than in both controls and the epineurial suture group, and this would be more consistent with the presence of recurrent sprouts. If this is the case, the presence of a small gap between the ends of the entubulated nerve stumps has not reduced the likelihood of nerve sprouts becoming recurrent.

Distal to the repair sites, the size of the myelinated axons and the myelin sheath thickness was reduced in both groups, and this is a well-recognized change after injury consistent with a reduction in conduction velocity (Aitken and Thomas, 1962; Orgel and Terzis, 1977; Smith and Robinson, 1995a,b). There was a reduction in the number of myelinated axons distal to entubulation but not distal to epineurial suture, and, although this suggests a better level of recovery in the suture group, we do not know the extent of branching at the injury site. There is a substantial increase in the number of myelinated axons distal to transection sites in rat sciatic nerves 60 days after injury (Beggs et al., 1986). It seems likely that the changes are related to time and that the timescale may differ for different repair procedures. A comparison of myelinated axon dimensions at 4 and 12 months after injury followed by epineurial suture repair shows a substantial shift toward the larger sizes (Hudson et al., 1979). A similar shift between 2 and 10 month has been reported following entubulation repair (Fields and Ellisman, 1986). The decline in myelin sheath thickness found in this report is similar to that which we and others have reported in regenerating fibers (e.g., Sanders, 1948; Holland et al., 1996).

The population of nonmyelinated axons distal to the repair site was greatly increased in both groups, and this increase was greater (although not statistically significantly so) after entubulation than after suture repair. In addition, the number of axons per Schwann cell was greater after entubulation repair but, as others have reported (Orgel and Terzis, 1977), was unchanged after epineurial suturing. Our previous electrophysiological studies on the efficacy of lingual nerve repair by these two methods revealed that more gustatory and thermosensitive fibers recovered after epineurial suture and the receptor characteristics of these fibers and the mechanosensitive afferents were nearer 
normal than after entubulation (Smith and Robinson, $1995 \mathrm{a}, \mathrm{b})$. In general, the present morphological data are consistent with our previous results and also indicate better recovery after epineurial suture repair. However, we would have expected the poorer recovery of gustatory and thermosensitive fibers after entubulation to be matched by a selective loss of small myelinated fibers proximal to the injury, as previously reported after unrepaired nerve section (Holland et al., 1996). The difference in functional recovery between the two procedures would seem to be due to the more extensive changes distal to the injury site after entubulation, and we were surprised by the large number of fibers distal to the entubulation repair.

The small number of animals included in this study restricts the effectiveness of statistical analysis in determining the significance of the changes that were measured. Smaller numerical differences that could be biologically significant may be statistically confirmed only by a much larger group of animals.

Direct comparison with other morphological studies after entubulation is difficult, since the variety of materials used to form tubes is wide and since other variables, such as the presence of neurotrophic substances, are often added. Some comparisons of entubulation and suture repair (e.g., Gibby et al., 1983) have concluded that entubulation is the more effective procedure, whereas others report no difference (Rosen et al., 1989). Merle et al. (1989) suggested that silicon entubulation may produce chronic nerve compression and lead to possible complications, but in our study, the internal diameter of the tube was significantly larger than the diameter of the nerve. No other studies have looked at these repair techniques on the lingual nerve with its varied and special populations of small fibers. It seems reasonable to suggest that there is no universally advantageous method of peripheral nerve repair and that the optimal technique will vary with the site and nature of the injury and the composition of the injured nerve. We (Smith and Robinson, $1995 \mathrm{a}, \mathrm{b})$ have previously speculated that the less effective recovery after entubulation may be due to the 1-mm gap that was left between the cut ends of the nerves, and it is possible that there may be less difference between the two groups after a longer period of recovery. Clinically, this gap may often be present following resection of a transected nerve or excision of a neuroma, and a bridging material may be needed. Tubes are among options that include grafts and a variety of resorbable materials. Tubes have a major advantage in providing a reservoir in which neurotrophic substances may be placed, should these prove effective in enhancing regeneration. These considerations will be included in future studies. Based on present findings, we conclude that epineurial suture is the preferred method for lingual nerve repair.

\section{Acknowledgments}

This study was supported by the Medical Research Council of Canada and the Wellcome Foundation.

\section{References}

Aitken JT (1949). The effect of peripheral connexions on the maturation of regenerating nerve fibres.J Anat 83:32-43.

Aitken JT, Thomas PK (1962). Retrograde changes in fibre size following nerve section. J Anat 96:121-129.

Ashur H, Vilner $Y$, Finsterbush A, Rousso M, Weinberg H, Devor M (1987). Extent of fiber regeneration after peripheral nerve repair: silicone splint vs. suture, gap repair vs. graft. Exp Neurol 97:365-374.

Beggs JL, Fischer DW, Shetter AG (1986). Comparative study of rat sciatic nerve microepineurial anastomoses made with carbon dioxide laser and suture techniques: Part 2. Neurosurg 18:266-269.

Blackburn CW, Bramley PA (1989). Lingual nerve damage associated with the removal of lower third molars. $\mathrm{Br}$ Dent J 167:103-107.

Cabaud HE, Rodkey WG, McCarroll HR Jr, Mutz SB, Niebauer JJ (1976). Epineurial and perineurial fascicular nerve repairs: A critical comparison. J Hand Surg 1:131-137.

Fields RD, Ellisman MH (1986). Axons regenerated through silicone tube splices. II. Functional morphology. Exp Neurol 92:61-74.

Fields RD, Le Beau JM, Longo FM, Ellisman MH (1989). Nerve regeneration through artificial tubular implants. Prog Neurobiol 33:87-134.

Gibby WA, Koerber HR, Horch KW (1983). A quantitative evaluation of suture and tubulisation nerve repair techniques. J Neurosurg 58:574-579.

Holland GR, Robinson PP (1990). The number and size of axons central and peripheral to inferior alveolar nerve injuries in the cat. J Anat 173:129-137.

Holland GR, Robinson PP (1992). Axon populations in cat lingual and chorda tympani nerves. J Dent Res 71:1468-1472.

Holland GR, Robinson PP, Smith KG, Pehowich E (1996). A quantitative morphological study of the recovery of cat lingual nerves after transection or crushing. J Anat 188:289-297.

Hudson AR, Hunter D, Kline DG, Bratton BR (1979). Histological studies of experimental interfascicular graft repairs. J Neurosurg 51:333-340.

Jenq CB, Coggeshall RE (1985). Nerve regeneration through holey silicone tubes. Brain Res 361:233-241.

Lubinska L (1961). Demyelination and remyelination in the proximal parts of regenerated nerve fibers. J Comp Neurol

Table 5. The percentage change in axon numbers and sizes in comparison with contralateral controls after epineurial suture and entubulation repairs. Asterisks indicate values that are statistically significantly different from controls $(\mathrm{p}<0.05)$.

\begin{tabular}{l|c|c|c|c|c|c}
\hline & \multicolumn{2}{|c|}{ Proximal Chorda Tympani } & \multicolumn{2}{c|}{ Proximal Lingual } & \multicolumn{2}{c}{ Distal } \\
\cline { 2 - 7 } & Sutured & Entubulated & Sutured & Entubulated & Sutured & Entubulated \\
\hline Number of myelinated axons & -9.35 & -23.63 & $+29.71^{*}$ & -4.55 & -6.68 & $-19.04^{*}$ \\
Size of myelinated axons & +1.35 & +1.08 & +5.69 & -6.80 & $-27.68^{*}$ & $-32.81^{*}$ \\
Thickness of myelin sheath & -2.82 & -15.58 & +0.88 & -15.00 & $-33.00^{*}$ & $-37.38^{*}$ \\
Number of non-myelinated axons & +18.16 & +88.89 & +160.00 & +95.79 & $+83.83^{*}$ & $+214.80^{*}$ \\
Number of axons per fiber & +5.29 & +40.57 & -0.75 & +15.42 & -14.34 & +33.63 \\
\hline
\end{tabular}


117:275-289.

Mason DA (1988). Lingual nerve damage following lower third molar surgery. Int J Maxillofac Surg 17:290-294.

Mayhew TM, Sharma AK (1984). Sampling schemes for estimating nerve fibre size. I. Methods for nerve trunks of mixed fascicularity. J Anat 139:45-58.

Merle M, Dellon AL, Campbell JN, Chang PS (1989). Complications from silicon-polymer intubulation of nerves. Microsurg 10:130-133.

Morris JH, Hudson AR, Weddell G (1972). A study of degeneration and regeneration in the divided sciatic nerve based on electron micrscopy. IV. Changes in fascicular microtopography, perineurium and endoneurial fibroblasts. Z Zellforsch Mikrosk Anat 124:165-203.

Orgel MG, Terzis JK (1977). Epineurial vs. perineurial repair. Plast Reconstr Surg 60:80-91.

Robinson PP (1989). The reinnervation of the tongue and salivary glands after lingual nerve injuries in cats. Brain Res
483:259-271.

Robinson PP (1992). The effect of injury on the properties of afferent fibres in the lingual nerve. Br J Oral Maxillofac Surg 30:39-45.

Rosen JM, Pham HN, Hentz VR (1989). Fascicular tubulization: a comparison of experimental nerve repair techniques in the cat. Ann Plast Surg 22:467-478.

Sanders FK (1948). The thickness of the myelin sheaths of normal and regenerating peripheral nerve fibres. Proc $R$ Soc Lond 135(B):323-357.

Smith KG, Robinson PP (1995a). The reinnervation of the tongue and salivary glands after two methods of lingual nerve repair in the cat. Arch Oral Biol 40:373-383.

Smith KG, Robinson PP (1995b). An experimental study of lingual nerve repair using epineurial suture or entubulation. Br J Oral Maxillofac Surg 33: 211-219.

von Arx DP, Simpson MT (1989). The effect of dexamethasone on neuropraxia following third molar surgery. $\mathrm{Br}$ ) Oral Maxillofac Surg 27:477-480. 